\title{
TOROIDAL MHD STABILITY IN COMPLEX MAGNETIC CONFIGURATIONS
}

\author{
H. M. RizK and T. Kammash \\ Department of Nuclear Engineering, University of Michigan, Ann Arbor, Michigan 48109, U.S.A.
}

(Received 24 September 1976; and in revised form 24 February 1977)

\begin{abstract}
Mercier's localized perturbation criterion near the magnetic axis is examined for a particular type of toroidal configuration of non-planar magnetic axis of variable curvature and torsion with non-circular (ellipticaily and triangularly deformed) plasma cross section for the 'resonance case'. As an application, a numerical calculation is carried out for a class of equilibria that belongs to the above configuration with constant torsion. The result of this numerical calculation indicates that in the absence of longitudinal current, it is possible to have a domain of stability in a system with a horizontally elliptical (and triangular) deformation with an ellipticity corresponding to $(a=2 b)$ i.e. the minor axis is twice the major axis. In the presence of a longitudinal current, a domain of stability is also possible and it depends on the direction of the current. In addition it is shown that horizontally elliptical and triangular deformations are favourable for stability.
\end{abstract}

Several authors (Mercier, 1962; Bineau, 1962; Greene and Johnson, 1962; SOLOV'Ev, 1968) have obtained the criteria of stability for localized perturbations in toroidal traps using different methods. MrkHanovskri (1973) showed that Mercier's general geometrical criterion (MERCIER, 1962) is necessary and sufficient for the stability of a plasma against a particular class of local perturbations. For all other local perturbations, Mercier's criterion does not hold. MERCrer (1964) developed his criterion near the magnetic axis and showed that for a circular plasma cross section, (magnetic surfaces near the magnetic axis can only be circular or elliptic) there is no domain of stability in the absence of a longitudinal current. Solvey'ey (1968), SOLvev'ev et al. (1969) and Sirarkañov (1968) obtained the necessary and sufficient criterion near the magnetic axis-referred to as (MrkHAILovskn, 1973)-the necessary criterion for the local stability-and showed that stability is indeed possible in the absence of a longitudinal current in a plasma with an elliptical cross section. Luc et al. (1974) expressed Mercier's criterion near the magnetic axis for the resonance case and studied the possibility of the existence of a stable domain in the absence of a longitudinal current in an elliptical plasma with a non-planar magnetic axis.

In the present paper we analyse Mercier's criterion near the non-planar magnetic axis and study the resonance case for a class of toroidal plasma already studied by Rrzk and KamMASH (1975-hereafter referred to as I). The case of interest corresponds to a non-circular (elliptically and triangularly deformed) plasma cross section through which a weak longitudinal current with flat radial profile flows parallel or antiparallel to the magnetic axis. Of special interest also is the case with no longitudinal current.

In order to write an explicit expression for Mercier's general geometric criterion for stability we follow the procedure outlined in $\mathbf{I}$ and introduce the ellipticity $-1 \leq E_{m} \leq 1$ of the non-circular plasma cross section near the magnetic axis; the ratio $j_{s 0} / 2 B_{s 0}$ of the longitudinal current density to the magnetic field in the same region; the function $P_{0}{ }^{\prime}=\mathrm{d} P /\left.\mathrm{d} \psi\right|_{\Psi=0}$ which is the derivative near the magnetic axis of the pressure $P$ with respect to the poloidal flux function $\Psi$; the functions $\tilde{r}, \tilde{s}$ which give the corrections to the elliptical form of the magnetic 
surfaces near the magnetic axis; the $\mathrm{d}^{\prime}(s) / 2=(\mathrm{d} / \mathrm{d} s)(\mathrm{d}(s) / 2)$ at which the cross sections turn around the magnetic axis (here $s$ is the curvilinear distance along the magnetic axis); the curvature $1 / R(s)$ and the torsion $1 / T(s)$ of the non-planar magnetic axis; and the associated resonant coefficient, $A_{k_{0}}$, that corresponds to the index, $k_{0}$ of the Fourier expansion of the relative curvature of the magnetic axis (ShafRanov, 1964). This curvature is given by

where

$$
\frac{1}{R(s)}=\sum_{k=-\infty}^{\infty} A_{k} \mathrm{e}^{i \delta_{k}(s)}
$$

$$
\delta_{k}(s)=\frac{2 \pi k s}{L}-\frac{\alpha(L)}{L}+\alpha(s)+\phi_{k}, \quad \alpha(s)=\int_{0}^{s} \frac{\mathrm{d} s}{T(s)}
$$

and $L=\oint \mathrm{d} s$ is the total length of the magnetic axis. It is important to note at this point that (Luc et al., 1974; and I) there is one and only one coefficient $A_{k_{0}}$ that corresponds to the index $k_{0}$. This feature can be especially dominant in the equilibrium, classical diffusion and stability problems of this type of complex magnetic toroidal configurations.

Mercier's localized perturbation criterion given by equation (70) of (MERCIER, $1964)$ for the class of equilibria described by $\eta(s)\left(=\tanh ^{-1} E_{m}(s)\right)$, $\dot{j}_{s o}$ and $B_{s o}$ being constants, is

$$
\begin{aligned}
& \left(-P_{0}{ }^{\prime}\right)\left[-\frac{1}{\operatorname{ch}^{2} \eta}\left(\frac{j_{s 0}}{2 B_{s o}}\right)^{2}-\operatorname{th}^{2} \eta\left(\frac{\varepsilon_{t}}{a}\right)^{2}+\left(\operatorname{th} \eta\left\langle\frac{\cos \mathrm{d}(s)}{R^{2}(s)}\right\rangle-\left\langle\frac{1}{R^{2}(s)}\right\rangle\right)\right. \\
& \left.+3 \sqrt{B_{s o}} \text { th } \eta\left(\tilde{r} \mathrm{e}^{-\eta}\left\langle\frac{\cos (\mathrm{d}(s) / 2)}{R(s)}\right\rangle+\tilde{s} \mathrm{e}^{+\eta}\left\langle\frac{\sin (\mathrm{d}(s) / 2)}{R(s)}\right\rangle\right)+\frac{B_{s 0}{ }^{2}}{L \operatorname{ch} \eta} \operatorname{Re} \oint(P Z) \mathrm{d} s\right]>0
\end{aligned}
$$

where $\langle g(s)\rangle=(1 / L) \int_{0}^{L} \mathrm{~d} s g(s), \quad P=P_{1}-i P_{2} ;$ the function $\mathrm{d}(s) / 2\left(=\phi_{k_{0}}+\alpha(s)-\right.$ $\left.\varepsilon_{t} s / a\right)$ is the turning angle of the minor axis of the ellipse relative to the principal normal to the magnetic axis, $\varepsilon_{t}=-\varepsilon_{0} k_{t} ; k_{t}=k_{0}-\frac{\alpha(L)}{2 \pi}$. The parameter $\varepsilon_{0}=(2 \pi a / L)$ is the inverse of the aspect ratio, and $a$ is the characteristic dimension of the plasma cross section.

$$
\begin{array}{r}
P_{1}=\frac{2}{\sqrt{B_{s o}}} \frac{1}{(2 \operatorname{ch} \eta-\operatorname{sh} \eta)}\left\{-\left(\frac{j_{s o}}{2 B_{s o}}\right) \mathrm{e}^{\eta / 2}\left(\frac{\sin (d(s) / 2)}{R(s)}\right)+\frac{\left|c_{c o}\right| \sqrt{B_{s o}}}{4} \kappa_{1}\left(\frac{\mathrm{e}^{\eta / 2} \operatorname{sh}^{2}(\eta / 2)}{\operatorname{ch} \eta / 2)}\right)\right. \\
\left.+\frac{\operatorname{sh} \eta}{4}\left(\frac{\mathrm{e}^{-\eta / 2} \cos (\mathrm{d}(s) / 2)}{R(s)}\right)^{\prime}+\frac{5}{4} K(s) \operatorname{sh} \eta \mathrm{e}^{\eta / 2}\left(\frac{\sin (\mathrm{d}(s) / 2)}{R(s)}\right)\right\}, \quad(2) \\
P_{2}=\frac{2}{\sqrt{B_{\mathrm{so}}}} \frac{1}{(2 \operatorname{ch} \eta+\operatorname{sh} \eta)}\left\{\left(\frac{j_{s o}}{2 B_{s o}}\right) \mathrm{e}^{-\eta / 2}\left(\frac{\cos (\mathrm{d}(s) / 2)}{R(s)}\right)+\frac{\left|c_{c o}\right| \sqrt{B_{s o}}}{4} \kappa_{2}\left(\frac{\mathrm{e}^{-\eta / 2} \operatorname{sh}^{2}(\eta / 2)}{\operatorname{ch}(\eta / 2)}\right)\right. \\
\left.-\frac{\operatorname{sh} \eta}{4}\left(\frac{\mathrm{e}^{\eta / 2} \sin (\mathrm{d}(s) / 2)}{R(s)}\right)^{\prime}+\frac{5}{4} K(s) \operatorname{sh} \eta \mathrm{e}^{-\eta / 2}\left(\frac{\cos (\mathrm{d}(s) / 2)}{R(s)}\right)\right\}
\end{array}
$$

In the above expressions prime means the derivative with respect to $s$. The function $P_{0}^{\prime}$ is given by equation (8) of (I), and $Z(s)=z(s) / 4 P_{0}{ }^{\prime}=$ $\left(\kappa_{1}(s)+i \kappa_{2}(s)\right) / 4 P_{0}^{\prime}$ is the solution of equation (43) in MERCIER (1964), which is 
given by formula (59) in (I). The function $c_{c o} / 2 \pi\left(=-k_{0}+L K / 2 \pi\right)$ with $K=$ $\sqrt{\left(1-E_{m}{ }^{2}\right.}\left(j_{s o} / 2 B_{s o}-\varepsilon_{t} / a\right)$ is the rotational transform near the magnetic axis.

As may be seen (Luc et al., 1974), there are singularities in the function $Z(s)$ when the rotational transform $l_{c o} / 2 \pi$ near the magnetic axis has an integer value namely $L K / 2 \pi=l_{\text {res }}+\epsilon$ with $|\epsilon| \ll 1$. The interesting case is that of the resonance given by $L K / 2 \pi=l_{\text {res }}=0$ at which the coefficient $A_{k_{0}}$ becomes dominant. For this case, we can neglect all terms $l \neq 0$ and using $L K / 2 \pi \ll 1$ we get

$$
\begin{aligned}
\operatorname{Re} \oint(P Z) \mathrm{d} s & =\frac{A_{k_{0}} \mathrm{e}^{-\eta / 2}}{B_{s o}^{3 / 2} K} \int_{0}^{L} \mathrm{~d} s P_{2} \\
& =\frac{A_{k_{0}} \mathrm{e}^{-\eta / 2}}{B_{s o}^{3 / 2} K} \cdot \frac{2 \mathrm{e}^{-\eta / 2} L}{\sqrt{B_{s o}}(2 \operatorname{ch} \eta+\operatorname{sh} \eta)}\left\{\left(\frac{j_{s o}}{2 B_{s o}}\right)\left\langle\frac{\cos (\mathrm{d}(s) / 2)}{R(s)}\right\rangle\right. \\
& \left.+\frac{\left|c_{c o}\right| P_{0}^{\prime} A_{k_{0}}}{K B_{s o}} \cdot \frac{\mathrm{e}^{-\eta / 2} \operatorname{sh}^{2}(\eta / 2)}{\operatorname{ch}(\eta / 2)}+\frac{5}{4} K \operatorname{sh} \eta\left\langle\frac{\cos (\mathrm{d}(s) / 2)}{R(s)}\right\rangle\right\}
\end{aligned}
$$

The integrals in (1) and (4) are given by formulae (15) in (I). Substituting from (4) and the integrals in (I) into (1) consistent with $L K / 2 \pi \ll 1$, we can write the final form of this criterion as

$$
\begin{aligned}
\left(-P_{0}{ }^{\prime}\right)\left[\left(E_{m}{ }^{2}-1\right)\left(\frac{j_{s o}}{2 B_{s o}}\right)^{2}-\left(\frac{E_{m} \varepsilon_{t}}{a}\right)^{2}\right. & \\
& +\left(E_{m} \sum_{k} A_{k} A_{2 k_{0}-k} \cos \left(\phi_{k}-\phi_{2 k_{0}-k}-2 \phi_{k_{0}}\right)-\sum_{k}\left|A_{k}\right|^{2}\right) \\
+ & 3 \sqrt{\bar{B}_{s o}} \tilde{r} E_{m} \sqrt{\frac{1-E_{m o}}{1+E_{m}}} A_{k_{0}}+\frac{2 A_{k_{0}}{ }^{2}\left(1-E_{m}\right)}{\left(j_{s o} / 2 B_{s o}-\varepsilon_{t} / a\right)\left(2+E_{m}\right)}\left(\frac{j_{s o}}{2 B_{s o}}\left(2+\frac{5}{4} E_{m}\right)-\frac{5}{4} \frac{\varepsilon_{t} E_{m}}{\alpha}\right) \\
& \left.+\frac{2 A_{k_{0}}{ }^{2}\left|c_{c o}\right|}{B_{s o}\left(j_{s o} / 2 B_{s o}-\varepsilon_{t} / a\right) c_{c o}} \frac{\mathrm{d} P_{(F)}}{\mathrm{d} F} \frac{\sqrt{1-E_{m}{ }^{2}}}{4} \mathscr{F}\left(E_{m}\right)\right]>0
\end{aligned}
$$

where

$$
\begin{aligned}
\mathscr{F}\left(E_{m}\right) & =\frac{4 \mathrm{e}^{-3 \pi / 2} \operatorname{sh}^{2}(\eta / 2) \operatorname{ch} \eta}{\operatorname{ch}(\eta / 2)(2 \operatorname{ch} \eta+\operatorname{sh} \eta)} \\
& =\frac{4 E_{m}^{2} \sqrt{1-E_{m}^{2}}}{\left(1+E_{m}\right)\left(2+E_{m}\right)^{2}\left[1+\sqrt{1-E_{m}^{2}}-E_{m}^{2} /\left(2+E_{m}\right)\right]}
\end{aligned}
$$

It is clear that in the case $E_{m}=0$, the pressure dependent term in the stability criterion dropout i.e. the critical value of $\beta$ (the ratio of the kinetic to magnetic pressure) for stability appears only in the case of highly elliptical cross section. It is convenient to express this criterion in terms of the purely geometric part, $V_{0}^{\prime \prime}$, (the vacuum magnetic well function) of the function $V^{\prime \prime}=\mathrm{d}^{2} V / \mathrm{d} \bar{\psi}^{2}$ near the non-planar magnetic axis, where $V$ is the volume bounded by magnetic surface $F=$ constant, and $\bar{\psi}$ is the longitudinal magnetic fiux function. This quantity characterizes the curvature of a magnetic well when $V^{\prime \prime}<0$ or a magnetic hill when $V^{\prime \prime}>0$. By introducing the expression for $V_{0}$ " near the magnetic axis for the resonance case (which is given by formula (16) in (I)) into (5), we obtain Mercier's 
criterion in terms of the vacuum magnetic well function $V_{0}^{\prime \prime}$. It is

$$
\begin{aligned}
& \left(-P_{0}\right)\left[-\frac{\pi B_{s o}^{2} \sqrt{1-E_{m}^{2}}}{L} V_{0}^{\prime \prime}+\left(E_{m}^{2}-1\right)\left(\frac{j_{s o}}{2 B_{s o}}\right)^{2}\right. \\
& +\frac{3}{2}\left(E_{m} \sum A_{k} A_{2 k_{0}-k} \cos \left(\phi_{k}-\phi_{2 k_{0}-k}-2 \phi_{k_{0}}\right)\right. \\
& \left.-\sum_{k}\left|A_{k}\right|^{2}\right)+\frac{4\left(1-E_{m}\right)}{\left(2+E_{m}\right)} \alpha_{1} A_{k_{0}}+\frac{2 A_{k_{0}}{ }^{2}\left(1-E_{m}\right)}{\left(j_{s o} / 2 B_{s o}-\varepsilon_{t} / a\right)\left(2+E_{m}\right)} \\
& \left.\times\left(\frac{j_{s o}}{2 B_{s o}}\left(2+\frac{5}{4} E_{m}\right)-\frac{5}{4}\left(\frac{\varepsilon_{t} E_{m}}{a}\right)\right)+\frac{2 A_{k_{0}}{ }^{2}\left|c_{c o}\right|}{B_{s o}\left(j_{s o} / 2 B_{s o}-\varepsilon_{t} / \alpha\right) c_{c o}} \frac{\mathrm{d} P_{(F)}}{\mathrm{d} F} \frac{\sqrt{ } 1-E_{m}{ }^{2}}{4} \mathscr{F}\left(E_{m}\right)\right]>0
\end{aligned}
$$

Where the parameters $\alpha_{1}$ and $\alpha_{3}$ are related to the parameter $\tilde{r}$ by the relation (12) in (I). The two forms of the stability criterion given by (5) and (6) can respectively be written for the case of axisymmetric Tokamak by setting $L=2 \pi R$, $k_{0}=0, A_{k_{0}}=1 / R, 1 / T=0$.

The MHD equilibrium problem for non-circular (elliptically and triangularly deformed) plasma in magnetic toroidal configuration with non-planar magnetic axis with variable torsion and curvature has been solved in (I) for weak current with flat profile flowing in both the forward and backward directions. This equilibrium solution in an $(x, y)$ coordinate system is written as:

with

$$
F=F_{s} F_{c}
$$

$$
\frac{F_{s}}{F_{0}}=1-X^{2}-Y^{2}\left(\frac{1-E}{1+E}\right)-\varepsilon_{s}\left[X^{3}-3 X Y^{2}\left(\frac{1-E}{1+E}\right)\right] \text {, }
$$

and

$$
F_{c}=1-\nu X+\varepsilon_{k} \nu\left[\gamma_{1} X^{2}+\gamma_{2} Y^{2}\left(\frac{1-E}{1+E}\right)\right]+\ldots
$$

where $X=x / a, Y=y / a$ and $F_{0}, \nu, \gamma_{1}, \gamma_{2}$ are constants that are given in details in (I). $\varepsilon_{s}$ is the appropriate distortion factor, $-1 \leq E \leq 1$ is the ellipticity parameter of the (boundary) magnetic surface. To obtain the above result, it was assumed that $P(F)=0, F=0$ on the toroidal surface of the triangularly deformed cross section given by $F_{s}=0$. Also, we have assumed that $\varepsilon_{k}\left(=a A_{k_{s}}\right) \sim$ $\varepsilon_{0} \sim \varepsilon_{s} \sim \varepsilon \ll 1$, the so-called 'thin-tube' approximation. The limiting value of $\beta$ for equilibrium (corresponding to the appearance of a new magnetic axis) has also been derived.

We now apply the stability condition given by (5) to this equilibrium by substituting from (38)-(40), (43) and (44) in (I) into (5). The result assumes the form

$$
\begin{gathered}
\left(-P_{0}\right)\left[\frac{\varepsilon_{0}{ }^{2}\left(E_{m}^{2}-1\right)}{a^{2} q_{s}^{2}}-\left(\frac{E_{m} \varepsilon_{t}}{a}\right)^{2}+\left(E_{m} \sum_{k} A_{k} A_{2 k_{0}-k} \cos \left(\phi_{k}-\phi_{2 k_{0}-k}-2 \phi_{k_{0}}\right)\right.\right. \\
\left.-\sum_{k}\left|A_{k}\right|^{2}\right)+\frac{3 A_{k_{0}}\left(M_{2} N_{2}-M_{1} N_{1}\right) E_{m}\left(1-E_{m}\right)}{2 a M_{1} M_{2}\left(2+E_{m}\right)} \\
\left.-\frac{4 A_{k_{0}}{ }^{2}}{G_{s}}\left(\frac{1}{q_{s}}-\frac{5}{8} E_{m} G_{s}\right)\left(\frac{1-E_{m}}{2+E_{m}}\right)-\frac{A_{k_{0}}{ }^{2}\left|c_{c o}\right| \beta \sqrt{1-E_{m}{ }^{2}}}{c_{c o} \varepsilon_{0}{ }^{2} G_{0} G_{s}\left(1+\varepsilon_{k} x_{m} / a\right)\left(1+E_{m}\right)} \mathscr{F}\left(E_{m}\right)\right]>0
\end{gathered}
$$


where

$$
\begin{aligned}
& G_{s}=-\left(\frac{1}{q_{s}}+k_{t}\right)=-G_{0}\left(\beta^{*} W-1\right) ; \quad G_{0}=-\left(\frac{1}{q_{0}}+k_{t}\right) ; \\
& \frac{1}{q_{s}}=\frac{1}{q_{0}}+\beta^{*} G_{0} W ; \quad \frac{1}{q_{s}}=\frac{a j_{s o}}{2 \varepsilon_{0} B_{s o}} ; \quad \frac{1}{q_{0}}=\frac{a j_{0}}{2 \varepsilon_{0} B_{0}} ; \\
& W=2 \varepsilon_{k}\left(\frac{x_{m}}{a}\right)-\left(\varepsilon_{k}{ }^{2}+\varepsilon_{t}^{2}\right)\left(\frac{x_{m}}{a}\right)^{2} ; \quad \beta^{*}=\frac{\beta}{\varepsilon_{0}{ }^{2} G_{0}^{2}(1+E)}
\end{aligned}
$$

$j_{0}$ and $B_{0}$ are respectively the central longitudinal components of the current density and magnetic field, and $\left(x_{m}, 0\right)$ are coordinate of the magnetic axis relative to the central axis. For low values of $\beta$ (for which $\beta^{*} \leqslant 1 ; 1 / q_{s} \sim 1 / q_{0} ; G_{s} \sim G_{0}$; $E_{m} \sim E ; x_{m} / a \sim \varepsilon ; \nu \sim \varepsilon ; M_{1}=M_{2} \simeq-1 ; N_{1} \simeq\left(\nu+3 \nu \varepsilon_{s}\right), N_{2} \simeq\left(\nu-\varepsilon_{s}\right)$ the criteria of stability (10) takes on the forms

$$
\begin{aligned}
\left(-P_{0}{ }^{\prime}\right)\left\{\frac{\varepsilon_{0}{ }^{2}\left(E^{2}-1\right)}{a^{2} q_{0}{ }^{2}}\right. & -\left(\frac{E \varepsilon_{t}}{a}\right)^{2}+\left(E_{m} \sum_{k} A_{k} A_{2 k_{0}-k} \cos \left(\phi_{k}-\phi_{2 k_{0}-k}-2 \phi_{k_{0}}\right)\right. \\
& \left.-\sum_{k}\left|A_{k}\right|^{2}\right)+\frac{2 A_{k_{0}}(1-E)}{(2+E)}\left[\frac{3 \varepsilon_{s} E}{\alpha}-\frac{2 A_{k_{0}}}{G_{0}}\left(\frac{1}{q_{0}}-\frac{5}{8} E G_{0}\right)\right] \\
& \left.-\frac{A_{k_{0}}{ }^{2}\left|c_{c o}\right| \beta \sqrt{1-E^{2}} \tilde{f}\left(E_{m}\right)}{\varepsilon_{0}{ }^{2} G_{0}{ }^{2} c_{c o}\left(1+\varepsilon_{k} x_{m} / a\right)(1+E)}\right\}>0 .
\end{aligned}
$$

It is clear from $(10)$ and $(11)$ that at $1 / a_{0}=-k_{i}$ (corresponding to resonance case) $G_{0}=0$ and the limiting value of $\beta$ for stability is zero.

In order to carry out numerical calculations for this criterion one needs first to calculate the Fourier components that represent the relative curvature. Unfortunately, it is not easy to find a non-planar closed curve with variable curvatire, and torsion. Attempts have been made by LuC et al. (1974); and RuzK and KAMMASH (1975) successfully in calculating a closed curve of variable curvature but with constant torsion. For our purposes here we find it sufficiently adequate to consider a closed, non-planar magnetic axis of variable curvature and constant torsion characterized by $(L / 2 \pi T)=2.84549$ and a resonance index of $k_{0}=3$. We find for this curve that $\sum_{k}\left|A_{k}\right|^{2}=0.30885, \sum_{k} A_{k} A_{2 k_{0}-k} \cos \left(\phi_{k}-\phi_{2 k_{0}-k}-2 \phi_{k_{0}}\right)=$ 0.1526 and $A_{k_{0}}=0.36413$. The properties of this curve have been detailed in (I) where it was shown (for non-circular cross sections) that the principal normal to the magnetic axis rotates $k_{0}$ times around its axis but in the opposite direction to that of the external magnetic surfaces. For circular cross sections such rotation is not very important since the magnetic surfaces are approximately concentric circles. With the above values we calculate the criterion for all possible shapes of the plasma cross section (circular, elliptic and triangularly deformed) in this class of equilibria i.e. with different values of $E$ and $\varepsilon_{s}$. We choose the inverse aspect ratio to be $\varepsilon_{0}=0.1$. Figures 1 and 2 show plots of $1 / q_{0}$ versus the limiting values of $\beta$ for the equilibrium calculated from equation (50) in (I). These are shown by the solid curves while those for stability as calculated from (10) are indicated by the broken curves. 


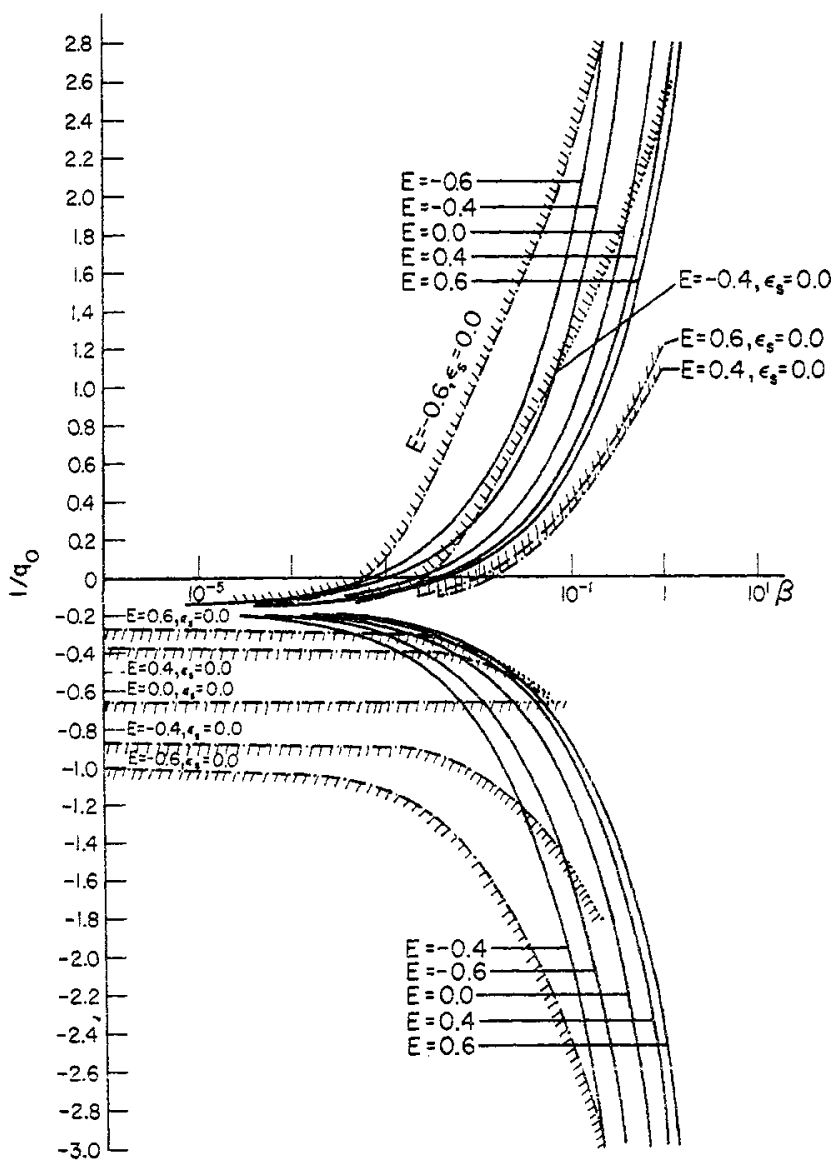

FIG. 1.-Domains of equilibrium and localized stability for an elliptical plasma cross section.

We observe for equilibrium that the influence of triangular deformation on the limiting value of $\beta$ is weak and can be neglected; and that a vertically elongated elliptic plasma cross section is preferable for equilibrium. Also we note that in the absence of a longitudinal current, equilibrium does not exist for high values of $\beta$ at which a new magnetic axis is supposed to appear. It does, however, exist for low values of $\beta$ (in the range $0-1.4 \times 10^{-5}$ for $E=-0.99$; and $0-7.05 \times 10^{-3}$ for $E=0.99$ ).

We turn now to consideration of the regions of stability for localized perturbations. The intersection of curves limiting the domain of equilibrium (as $\nu$ approaches 1 ) and stability gives the limiting value of $\beta$ for each magnetic surface. The domains of stability are larger when the sign of $\varepsilon_{s}$ is the same as that of $E$.

In the absence of a longitudinal current, $1 / q_{0}=0$ we see from Figs $1-3$ that there is a small domain of stability for a horizontally elliptic cross section beginning only with an ellipticity of $E=-0.6$. This corresponds to $a=2 b$ at which $\beta_{P_{\max }}$ drops to about one third of its value for a circular cross section. The relative change in the average diffusion velocity $\Delta V_{D} / V_{s}=\left(\left\langle V_{D}\right\rangle / V_{s}-1\right)$ given in (I) increases by a factor of 2.5 of its value for circular cross section. This domain 


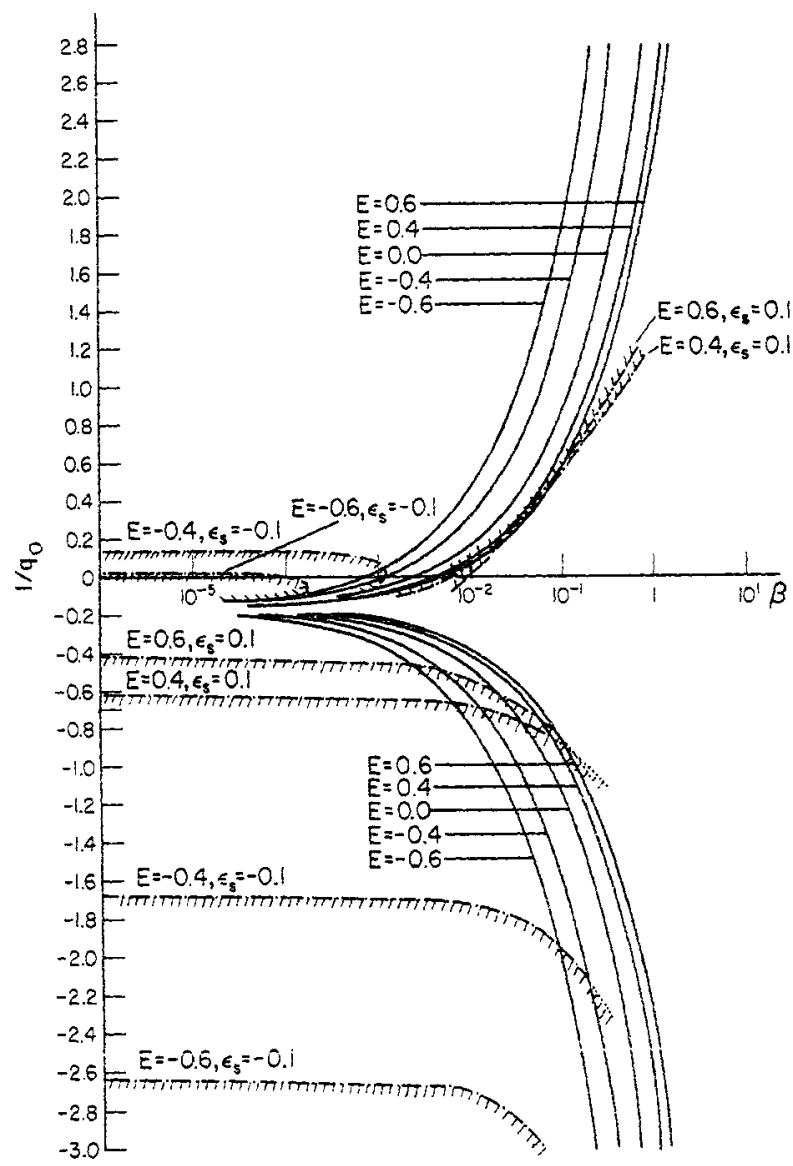

Frg. 2.-Domains of equilibrium and localized stability for plasma cross section with triangular deformation.

increases with decreasing ellipticity. For triangular deformations a domain of stability appears only at a horizontal deformation corresponding to $E=-0.6$ and $\varepsilon_{s}=-0.1$ and this domain increases with decreasing ellipticity also. We notice that the domain of stability with a horizontally triangular deformation is larger than that in the case of a horizontally elliptic cross section.

In the presence of a longitudinal current it is clear from Figs 1 and 2 that the domains of stability depend on the directions of the current (i.e. the sign of $1 / q_{0}$ ). We note that there is absolutely no domain for equilibrium or stability for the resonance case, $1 / q_{0}=-k_{\mathrm{t}}=-0.1545$ at which $G_{0}=0$ and $\beta=0$. In the region $-k_{1}<1 / q_{0}<0$ there is a weak stabilization current with horizontally elliptic and triangular cross sections for which $E=-0.6$, and $E=-0.6$ with $\varepsilon_{s}=-0.1$ respectively. In the case of a current flowing in the forward direction $\left(1 / q_{0}>-k_{t}\right)$ there is no domain of stability for circular $\left(\varepsilon_{s}=0, E=0\right)$, vertically elliptical $\left(\varepsilon_{s}=0\right.$, $E>0)$ or vertically triangular $\left(\varepsilon_{s}>0, E>0\right)$ deformations. There exists however a small domain of stability that increases with decreasing ellipticity for horizontally elliptical $\left(\varepsilon_{s}=0, E<0\right)$ cross sections. This domain is smaller than the corresponding case of the horizontally deformed triangular cross section. In the case of 


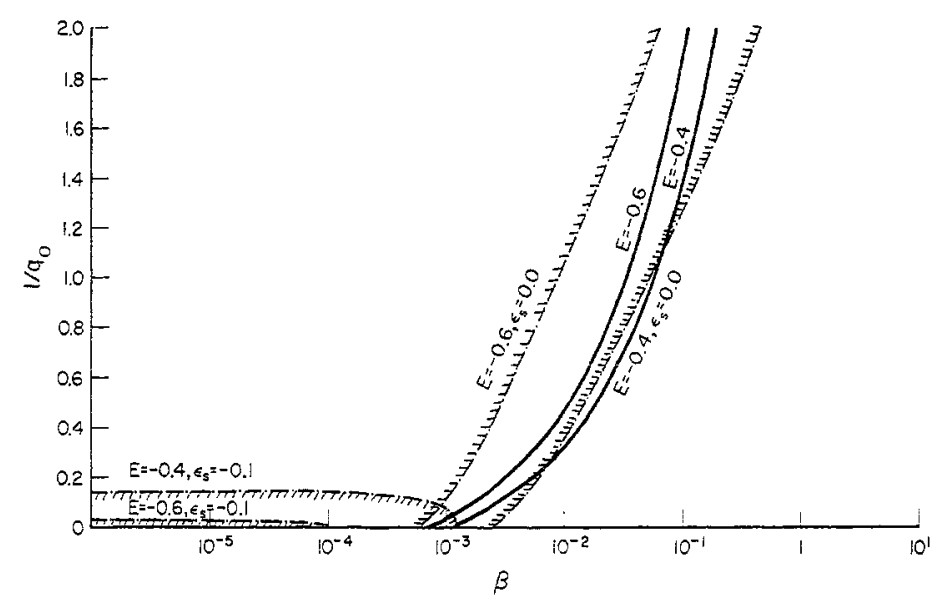

FIG. 3.-Domains of equilibrium and stability for elliptically and triangularly deformed plasma cross sections in the absence of longitudinal current.

a backward flowing current $\left(1 / q_{0}<-k_{t}\right)$ a domain of stability exists for all possible shapes of magnetic surfaces.

In conclusion, we note that for this type of toroidal plasma configuration with non-planar magnetic axis of variable curvature and a constant torsion of $L / 2 \pi T>$ 0 , in a vacuum magnetic well, equilibrium is possible in the absence of a longitudinal current at low values of $\beta$. The effect of triangular deformation on the limiting value of $\beta$ for equilibrium is weak and can be neglected. We also observe that a vertically elongated elliptical plasma cross section is favorable for equilibrium. Stability is indeed possible in the absence of longitudinal currents for only horizontally elliptical and triangular deformations with ellipticity corresponding to $a=2 b$ at low $\beta$. Moreover, it should be noted that a longitudinal current in the forward direction may be allowed to flow in horizontally elliptically or triangularly deformed cross sections without jeopardizing equilibrium or stability. In such systems most of the stabilization occurs when the current flows in the backward direction. Finally, the localized stability criterion indicates that horizontally elliptical and triangular deformations are preferable both in the presence and absence of a longitudinal current.

Acknowledgement-This work is supported by the U.S. Energy Research and Development Administration.

\section{REFERENCES}

Bineau B. (1962 Nucl. Fusion Suppl. 2, 130.

Greene J. M. and Johnson J. L. (1962) Physics Fluids 5, 510.

Luc H., Mashke E. K., Mercier C., Misguich J. H. and Rizk, H. M. (1974) Plasma Phys. 16, 544.

MERCIER C. (1962) Nucl. Fusion Suppl. 2, 801.

MERCIER C. (1963) Nucl. Fusion 3, 89.

MerCier C. (1964) Nucl. Fusion 4, 213.

MikhatrovsKn A. B. (1973) Soviet Phys. JETP 37, 274.

Rizk H. M. and Kammash T. (1975) Plasma Phys. 17, 1049.

Shafranov V. D. (1964) Nucl. Fusion 4, 232.

SHAFRANOV V. D. (1968) Proceedings of the First All Union Conference on Plasma Confinement in Closed Magnet Systems, Moscow, FIAN, 15-19 April. 
Shafranov V. D. and Yurchenko E. J. (1968) Nucl. Fusion 8, 329.

SOLOV'EV L. S. (1968) Soviet Phys. JETP 26, 400.

SOLOV'Ev L. S. (1968) Soviet Phys. JETP 26, 1167.

SOLOV'Ev L. S. (1969) Soviet Phys. Dokl. 13, 1050.

Solov'ev L. S., Shafranov V. D., Yurchenko E. J. (1969) Nucl. Fusion Suppl. 25.

Solov'ev L. S. and Shafranov V. D. (1970) Reviews of Plasma Physics (Edited by M. A. LeONTOVich) Vol. 5. Consultants Bureau, New York. 